\title{
A Comparative Study of Dirichlet and Neumann Conditions for Path Planning through Harmonic Functions
}

\author{
Madhuri Karnik, Bhaskar Dasgupta ${ }^{\star}$, and Vinayak Eswaran \\ Department of Mechanical Engineering, \\ Indian Institute of Technology, Kanpur - 208 016, India. \\ \{madhuri, dasgupta, eswar\}@iitk.ac.in
}

\begin{abstract}
Harmonic functions, by virtue of their extrema appearing only on the domain boundary, are known to have an advantage as a global potential function in the potential field based approach for robot path planning. However, a wide range of possibilities exist for the global harmonic function for a particular situation, depending on the boundary conditions. This paper conducts a comparison of two major classes of boundary conditions, namely Dirichlet and Neumann conditions, and attempts to discover their relative merits and demerits. It is found that the Neumann conditions offer a surer and faster approach to the path planning problem, though suffering from the disadvantage of occasional tendency of the planned path to graze along the domain boundary.
\end{abstract}

Keywords: Harmonic function, Motion planning, Global potential.

\section{Introduction}

Harmonic functions are solutions to Laplace's equation. They are known to have a number of properties useful in robotic applications (Connolly and Gruppen [1]). Harmonic functions offer a complete path planning algorithm and paths derived from them are generally smooth. When applied to path planning of robots, they have the advantage over simple potential field based approach, as they exhibit no spurious local minima.

The use of potential functions for robot path planning, as introduced by Khatib [2], views every obstacle to be exerting a repelling force on an end effector, while the goal exerts an attractive force. Koditschek [3], using geometrical arguments, showed that, at least in certain types of domains (like spherical domains with spherical obstacles), there exists potential functions which can guide the effector from almost any point (excepting a set of measure zero) to a given

\footnotetext{
* Author to whom all correspondence should be addressed at the current address: Technische Universität Berlin, Institut für Technische Informatik und Mikroelektronik, Sekretariat EN 10, Einsteinufer 17, D-10587 Berlin, Germany. Phone: ++49-30314-25324; Fax: ++49-30-314-21116; Email: dasgupta@cs.tu-berlin.de
} 
point. The usual formulation of potential fields for path planning does not prevent the spontaneous creation of local minima other than the goal. This may cause the robot to terminate its path at such a minimum and achieve a stable configuration short of goal.

Connolly et al [4] and Akishita et al [5] independently developed a global method using solutions to Laplace's equations for path planning to generate a smooth, collision-free path. The potential field is computed in a global manner, i.e. over the entire region, and the harmonic solutions to Laplace's equation are used to find the path lines for a robot to move from the start point to the goal point. Obstacles are considered as current sources and the goal is considered to be the sink, with the lowest assigned potential value. This amounts to using Dirichlet boundary conditions. Then, following the current lines, i.e. performing the steepest descent on the potential field, a succession of points with lower potential values leading to the point with least potential (goal) is found out. It is observed by Connolly et al [4] that this process guarantees a path to the goal without encountering local minima and successfully avoiding any obstacle, as a harmonic function cannot possess an extremum value except at the domain boundary.

In this paper, an attempt has been made to follow the above paradigm for path planning, using the analogy of temperature and heat flux for the potential and path line, respectively, for characterizing the solution of the Laplace equation. The analysis is carried out on two-dimensional domains having square or rectangular outer boundaries with occasional convolutions and obstacles. The finite element program "NASTRAN" is used to solve the Laplace equation and to obtain the temperature values at each node. Considering the robot to be a point, paths based on the potential (i.e. temperature) values are investigated by applying two types of boundary conditions. For Neumann boundary conditions, the start point is taken at a high temperature and the goal point is taken at a lower temperature, while the outer and the inner (obstacle) boundaries are treated as "No flux" (Homogeneous Neumann) boundaries. For Dirichlet boundary conditions, boundaries and the start point are assigned higher temperatures relative to the goal point. Various configurations and dimensions of the obstacles have been tried, with various temperature values assigned to them. The results obtained are analysed for colision-free path of the point robot.

\section{Harmonic Function}

A harmonic function on a domain $\Omega \subset R^{n}$ is a function which satisfies Laplace's equation,

$$
\nabla^{2} \Phi=\sum_{i=1}^{n} \frac{\partial^{2} \Phi}{\partial x_{i}^{2}}=0
$$

where $x_{i}$ is the $i$-th Cartesian coordinate and $n$ is the dimension. In the case of robot path construction, the boundary of $\Omega$ (denoted by $\partial \Omega$ ) consists of the outer boundary of the workspace and the boundaries of all the obstacles as well 
as the start point and the goal point, in a configuration space representation. The spontaneous creation of a false local minimum inside the region $\Omega$ is avoided if Laplace's equation is imposed as a constraint on the functions used, as the harmonic functions satisfy the min-max principle [6,7].

The gradient vector field of a harmonic function has a zero curl, and the function itself obeys the min-max principle. Hence the only types of critical points which can occur are saddle points. For a path-planning algorithm, an escape from such critical points can be found by performing a search in the neighbourhood of that point. Moreover, any perturbation of a path from such points results in a path which is smooth everywhere [1].

\section{Configuration Space}

In the framework used in the analysis here, the robot, i.e. its configuration, is represented by a point in the configuration space, or $\mathrm{C}$-space. The path planning problem is then posed as an obstacle avoidance problem for the point robot from the start point to the goal point in the $\mathrm{C}$ - space.

The C-space examples used in this paper have either square or rectangular outer boundaries, having projections or convolutions inside to act as barriers. Apart from projections of the boundaries, some obstacles inside the boundary are also considered. The $\mathrm{C}$-space is discretized and the coordinates and function values associated with each node are studied with the thermal analysis code of the finite element software NASTRAN (version 9.0). The highest temperature is assigned to the start point whereas the goal point is assigned the lowest for both Neumann and Dirichlet boundary conditions. In some cases with Dirichlet conditions, the start point is not assigned any temperature. The results are processed, for Dirichlet boundary conditions, by assigning different temperature values to the boundaries.

The area occupied by the obstacles, the temperatures or fluxes assigned to the boundaries and the goal points are the variables used to investigate the effectiveness of the technique in the present study.

\section{Boundary Conditions}

Solutions to the Laplace's equation were examined with two different types of boundary conditions. The following forms of the Dirichlet and Neumann boundary conditions were used.

\section{Dirichlet boundary conditions:}

$$
\Phi \mid \partial \Omega=c
$$

where $c$ is constant. In this case, the boundary is maintained at a particular temperature. The typical obstacle temperature values used are $8^{\circ} \mathrm{C}$ and $3^{\circ} \mathrm{C}$. The values of goal temperature used were $0^{\circ} \mathrm{C}$ and $-150^{\circ} \mathrm{C}^{1}$. If assigned, the

\footnotetext{
${ }^{1}$ This extremely low temperature is used to examine its effect on the results.
} 
start point is at $10^{\circ} \mathrm{C}$. As the boundary temperature is fixed, the heat flow has to be along the normal to the boundary.

\section{Homogeneous Neumann boundary conditions:}

$$
\frac{\partial \Phi}{\partial \bar{n}} \mid \partial \Omega=0
$$

where $\bar{n}$ is the unit vector normal to the boundary. This condition is applied only to the outer and the obstacle boundaries. The start point (the source) and the goal point (the sink) are maintained at $10^{\circ} \mathrm{C}$ and $0^{\circ} \mathrm{C}$ respectively (in the current implementation). The Neumann condition constrains the normal component of the gradient of $\Phi$ to be zero at the boundaries. As there is no normal component of heat flow, the condition forces the flow to be tangential to the boundary. As the path follows the heat flow, the Neumann condition may lead to a tendency for a robot to stay close to C-space obstacle/boundary surfaces.

\section{Path Planning}

Once the harmonic function under the boundary conditions is established, the required path can be traced by the steepest descent method, following the negative gradient from the start point through successive points with lower temperature till the goal, which is the point with the lowest temperature. The coordinates and the nodal gradients of temperature obtained from the finite element analysis can be used to draw the path.

In the present work, the actual path is not drawn, but the possibility and efficiency of tracing the path is investigated from the nature of the harmonic function in the domain. The presence of a significant temperature gradient all over the C-space indicates that a path can be traced for the point robot by the use of the gradient information. On the other hand, absence of an appreciable gradient, even in a portion of the $\mathrm{C}$-space, will prevent the efficient tracing of a path.

\section{Results and Discussion}

In this section, we present results for some simple C-space structures and compare the effects of various conditions on the contours of the resulting harmonic functions. These contours are enough to draw conclusions regarding the possibility and efficiency of developing the actual path from the function ${ }^{2}$. In the following, results of the Neumann condition are presented first, followed by solutions with Dirichlet conditions. The figures are drawn to show the geometry of the C-space and the temperature contours. The presence of temperature contours throughout the C-space indicates the presence of temperature gradient in

\footnotetext{
${ }^{2}$ The solution from the software also provides direct information of gradients, which are to be used for tracing the path. But, that data is not so visually revealing as the contours, hence have been omitted from presentation here.
} 
the C-space. This shows that the path can be easily and efficiently found using steepest descent method. The contours are labelled from 1 to B; 1 being the contour with the lowest temperature and $\mathrm{B}$ with the highest. The values of the minimum temperature (goal point) and the highest temperature (start point or boundary) and the node identities representing these points are also indicated on each figure. The computational time for both cases are the same, namely approximately 0.25 to $0.30 \mathrm{sec}$ of CPU time in a SUN workstation.

\subsection{Results with Neumann Boundary Condition}

Figures 1-3 show the temperature contours obtained by applying the Neumann boundary condition to the function in three test regions. The start point is maintained at $10^{\circ} \mathrm{C}$ and the goal point at $0^{\circ} \mathrm{C}$ in all the cases ${ }^{3}$. The effect of variation in the area and shapes of the obstacle boundaries on the gradient in the $\mathrm{C}$-space is examined. In the first case, the region is obtained by cutting off a convoluted corridor from a unit square. The convolution or inside projection from the boundary itself acts as a barrier and there is no true obstacle. In figure 2, there are two obstacles inside a (unit) square region, and the close proximity of the obstacles induces disturbance in the contour in the neighbourhood. In figure 3 , we have an example with both external boundary convolution and an obstacle in a rectangular region of $1.2 \times 1.0$ units. In all the figures, the origin is at the bottom left corner of the region, and the frame displayed inside shows only the directions.

It is observed that the temperature contours are well-spread in the region and a significant gradient is present throughout the C-space in each of the cases. Even at saddle points with zero gradient, one such possibility occurring between contour lines marked 'A' and ' 0 ' in figure 2, a slight perturbation from the point will succeed in resuming a fruitful path tracing. This is an advantage with the Neumann boundary conditions. On the other hand, motion orthogonal to the contour lines, as resulting from gradient-based motion planning, is likely to graze boundaries closely at times and even follow a longer path, rendering the method less than optimal. A large number of other examples tested show similar behaviour.

\subsection{Results with Dirichlet Boundary Condition}

Figures 4-7 show the temperature contours in the C-space when the Dirichlet boundary condition is applied to the function. All these results show a remarkable difference from the previous results. Here, the contour lines are mostly crowded near the points with assigned temperature values, leaving most of the free-space with very little temperature variation. Figure 4 shows the result with Dirichlet condition (with boundary temperature $3^{\circ} \mathrm{C}$ ) for the same region as in figure 1.

\footnotetext{
${ }^{3}$ Results are completely insensitive to these two temperature values (as long as they are different), as there is only one 'temperature difference' and its unit or scale is arbitrary.
} 


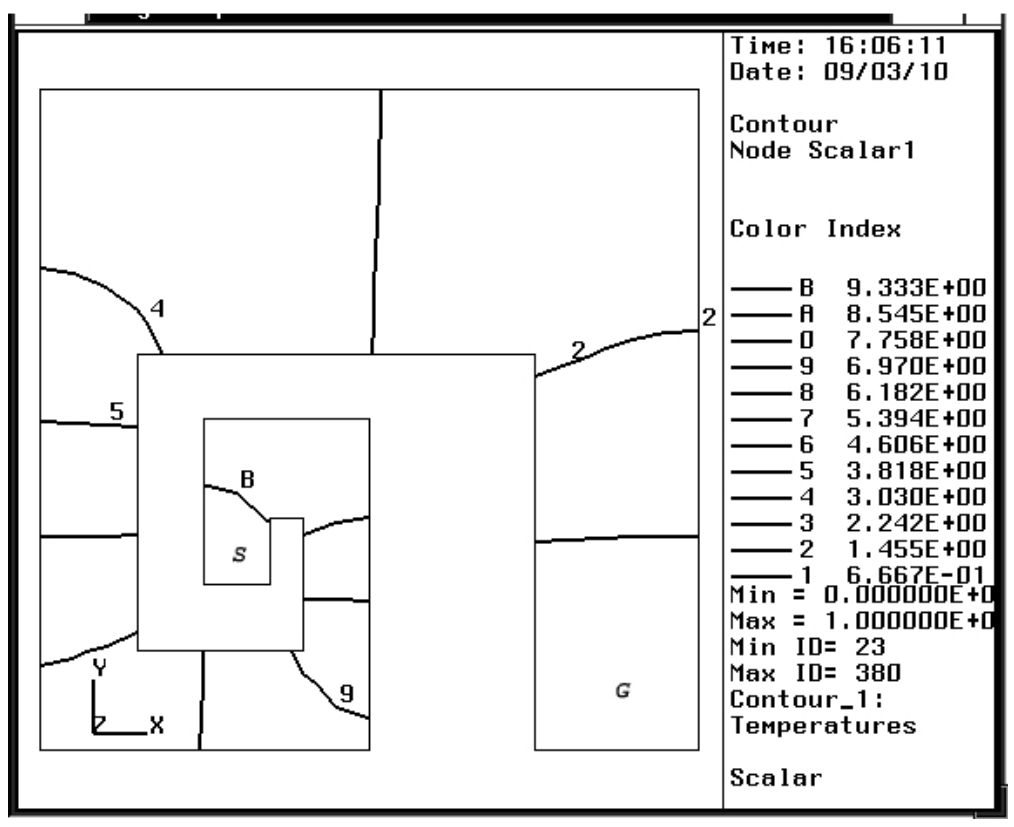

Start point: S $(0.3,0.3)$, Goal point: G $(0.9,0.1)$

Fig. 1. C-space with inside boundary projection (Neumann condition)

A comparison of the two figures shows the relative difficulty of path tracing through a gradient-based strategy in the case of the Dirichlet condition solution (figure 4). Changing the boundary temperature has little qualitative effect on the result. As figure 5 shows, the contour curves have got only a little redistributed, when we solve the same problem with boundary temperature maintained at $8^{\circ} \mathrm{C}$. Even modifying temperature difference drastically does not spread the contours well enough. For example, figure 6 shows the result of changing the goal point temperature to $-150^{\circ} \mathrm{C}$. From the result, we see that most of the contour curves are used up in accommodating the temperature difference between the boundary and the goal point, leaving most of the region essentially featureless (less than $1^{\circ} \mathrm{C}$ temperature difference between curves ' 0 ' and ' $\mathrm{A}$ '). Different sets of Dirichlet conditions applied to many other example regions, from simple to reasonably complicated, exhibit similar patterns of the contours.

One may notice, that in the case of Dirichlet condition, temperature values are assigned to the boundaries and, for obtaining a harmonic solution, it is not necessary to assign temperature values to both start and goal points. In figure 7 , we have an example (region the same as that of figure 3) with only two temperature values - boundary temperature $8^{\circ} \mathrm{C}$ and goal temperature $0^{\circ}$. The result, as expected, shows significant temperature gradient only close to the goal point. 


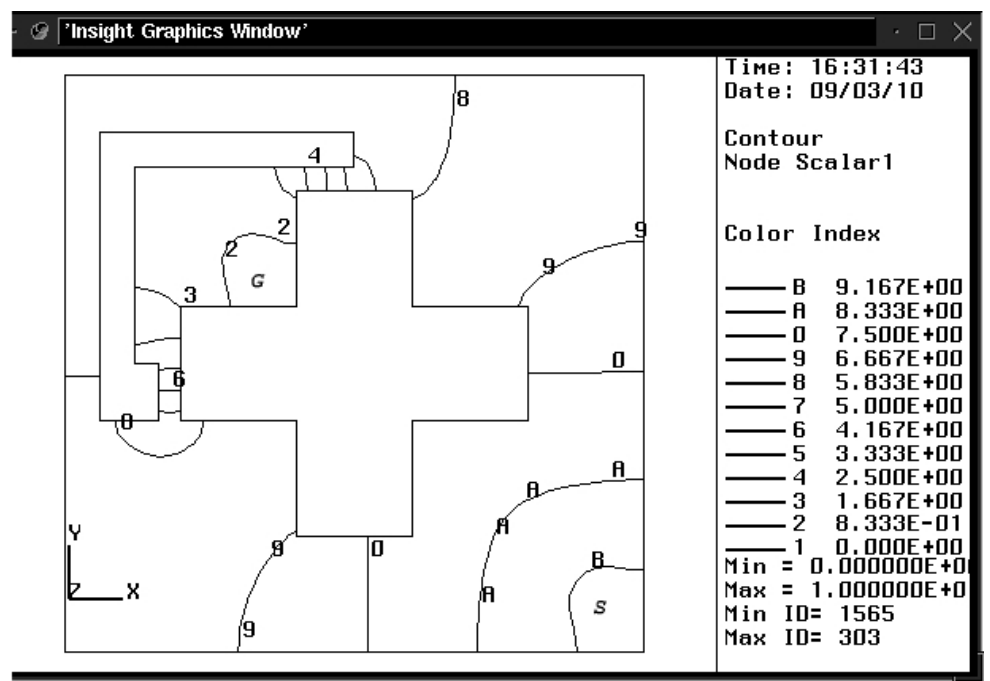

Start point: S $(0.9,0.1)$, Goal point: $\mathrm{G}(0.3,0.6)$

Fig. 2. C-space with obstacles (Neumann condition)

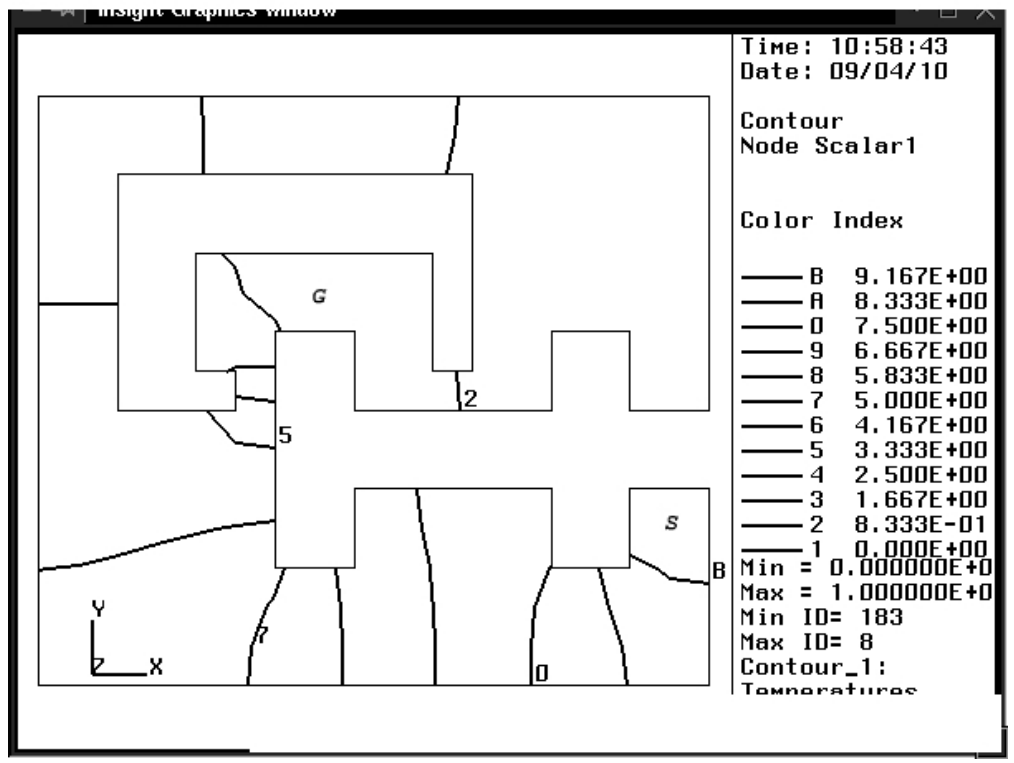

Start point: S $(1.1,0.3)$, Goal point: $\mathrm{G}(0.5,0.7)$

Fig. 3. Rectangular C-space with projection and obstacle (Neumann condition) 


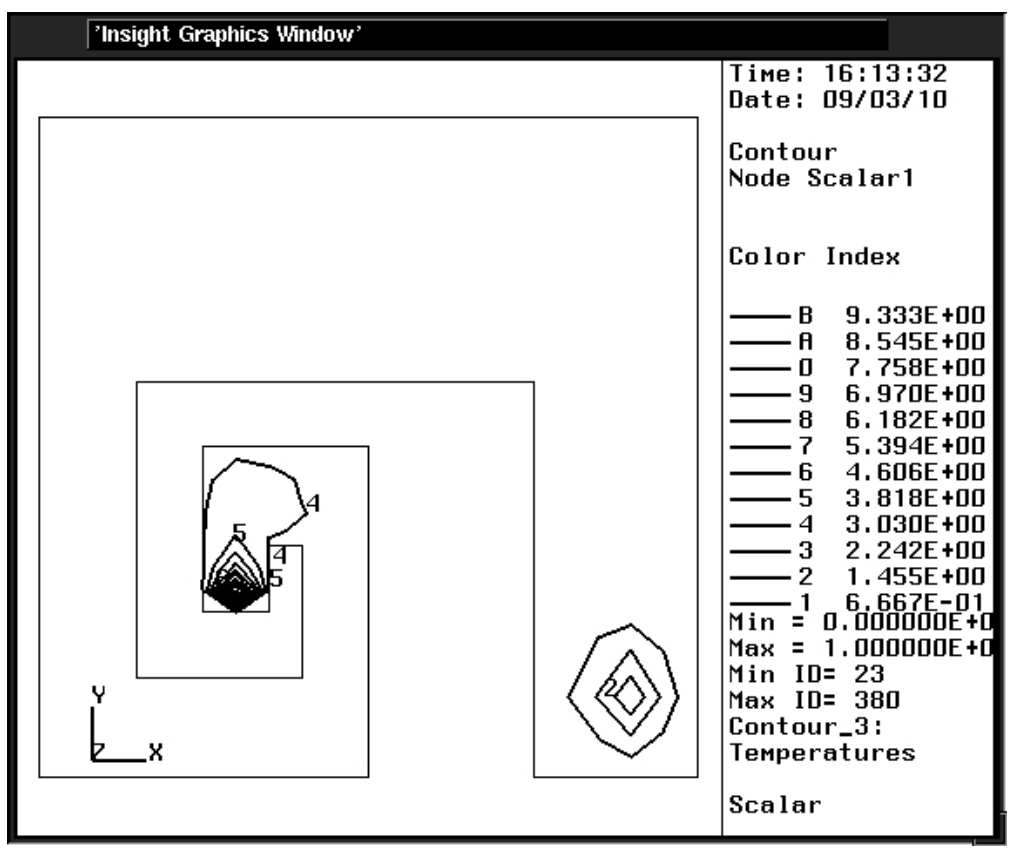

Start point: $(0.3,0.3)$, Goal point: $(0.9,0.1)$

Fig. 4. C-space with inside boundary projection (Dirichlet condition)

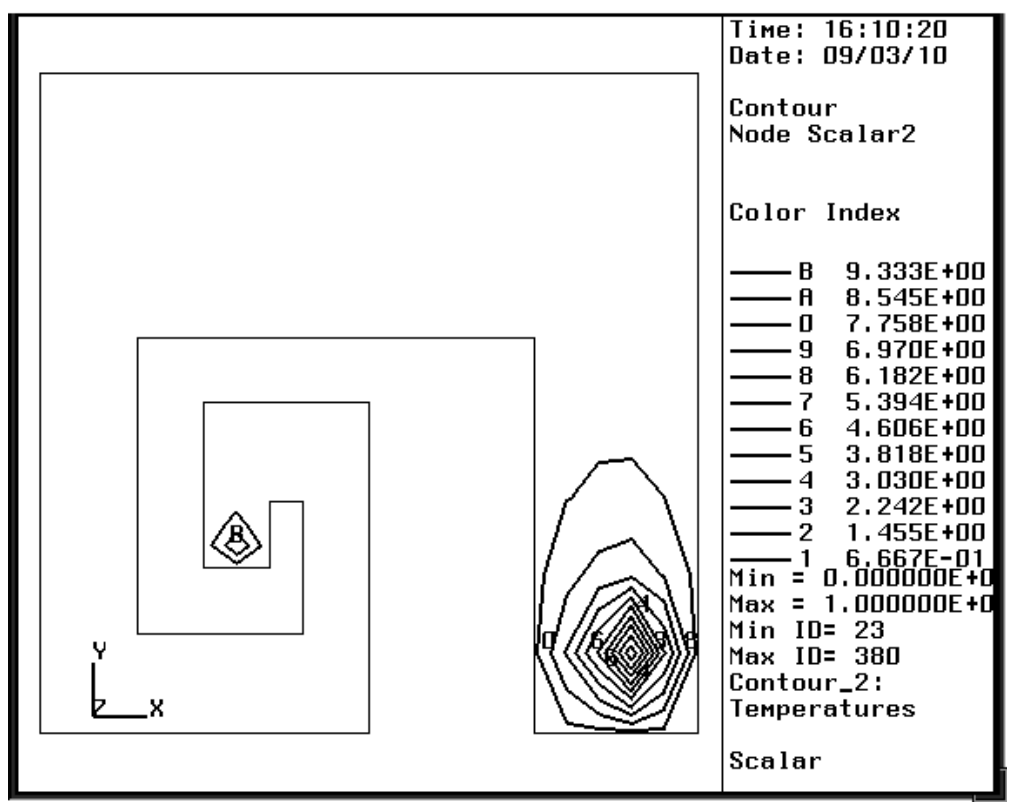

Start point: $(0.3,0.3)$, Goal point: $(0.9,0.1)$

Fig. 5. Effect of boundary temerature (Dirichlet condition) 


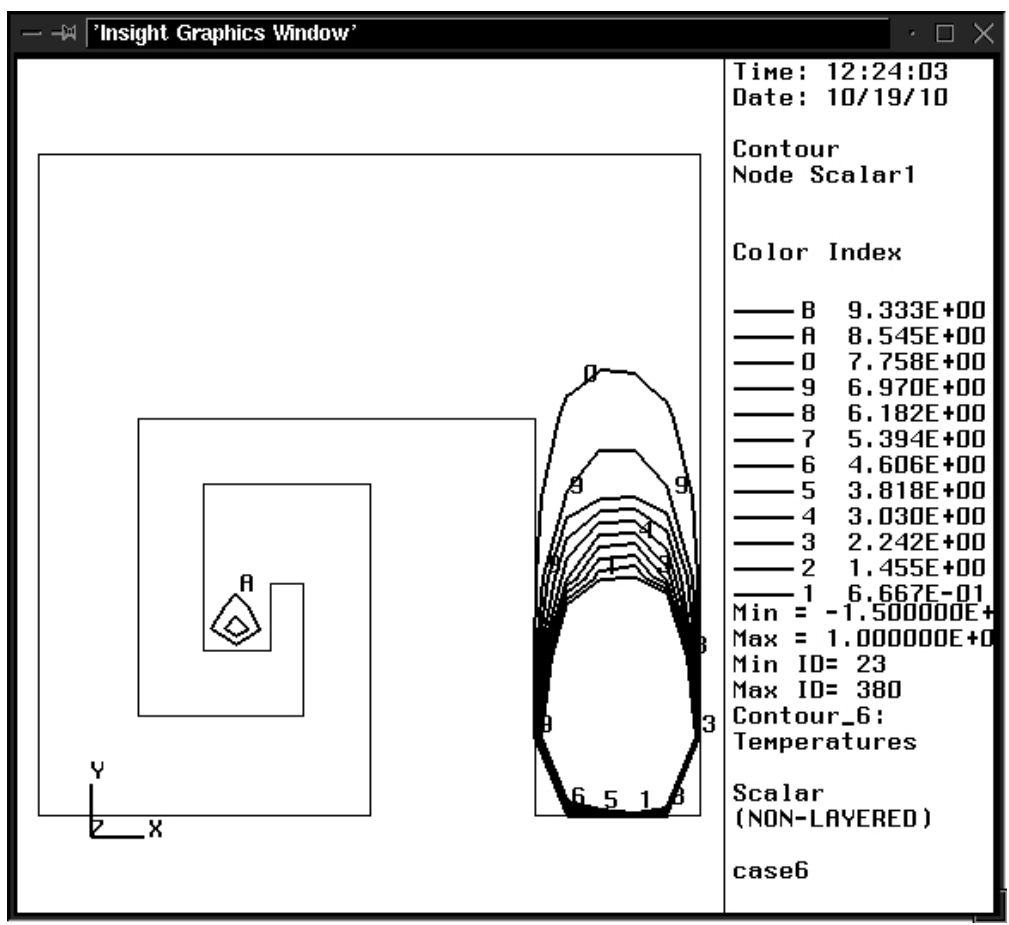

Start point: $(0.3,0.3)$, Goal point: $(0.9,0.1)$

Fig. 6. Effect of very low temperature at goal point (Dirichlet condition)

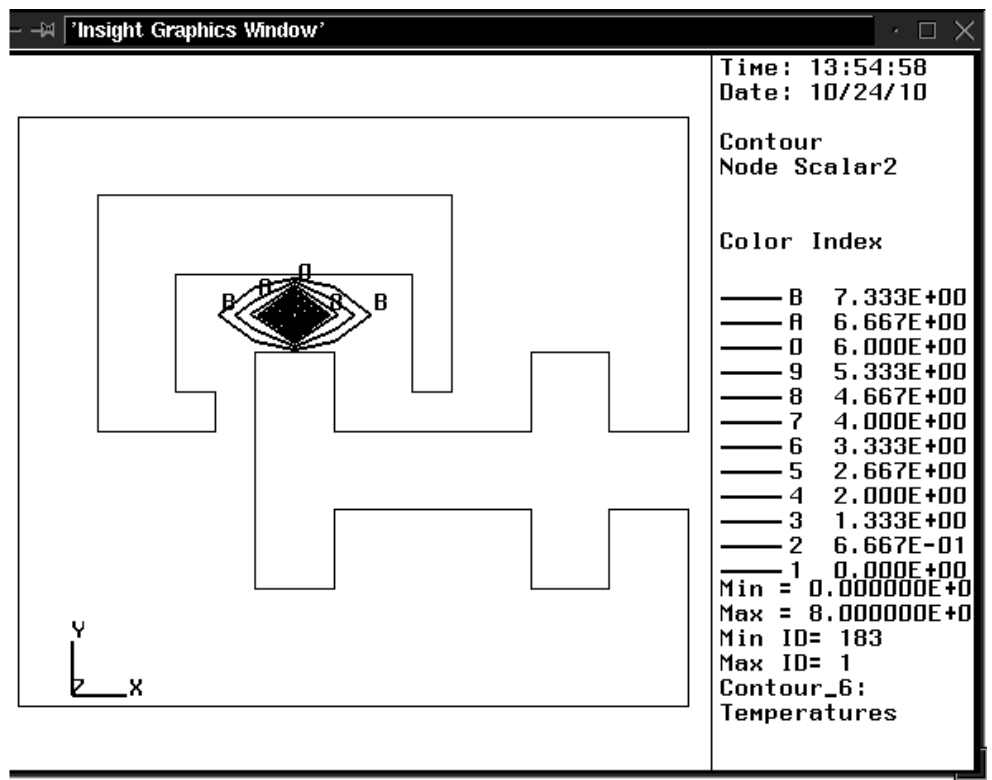

Start point not assigned, Goal point: $(0.5,0.7)$

Fig. 7. Solution with start point not assigned (Dirichlet condition) 


\subsection{Discussion}

The harmonic solutions presented above show that the two classes of boundary conditions (Neumann and Dirichlet) give quite different potential fields that can be used for path planning in different C-space configurations.

For all the C-spaces analysed with Neumann boundary condition, significant temperature gradient is present all over the $\mathrm{C}$-space making it easier to trace the path of the point robot. But the results obtained with Dirichlet boundary condition are radically different. The remarkable gradient is present only in the region close to the goal point (and also in the vicinity the start point, if it is assigned a temperature value), leaving the rest of the $\mathrm{C}$-space at (almost) constant temperature, with no appreciable gradient. Such a situation makes it difficult to trace a path to the goal point.

\section{Conclusions}

The comparative studies of Neumann and Dirichlet boundary conditions reveal that one has to judiciously choose the boundary conditions to be applied to Harmonic function used for robot path planning. The Neumann boundary condition gives the significant gradient of temperature (potential) throughout the $\mathrm{C}$-space irrespective of the shape of the obstacles and temperatures assigned to goal point and start point. This makes it an easier and surer method to follow the steepest descent for path planning. But the paths found out by this method may have a tendency to graze the boundary.

As extension of this work, the performance of the method in spaces of higher dimensions may be examined. Investigation of mixed boundary conditions is also an important area of possible further studies.

\section{References}

1. Connolly, C. I., Gruppen, R.: On the applications of harmonic functions to robotics. Journal of robotic systems. Journal of Robotic Systems 10 (7) (1993) 931-946

2. Khatib, O.: Real time obstacle avoidance for manipulators and mobile robots. IEEE Transactions on Robotics and Automation 1 (1985) 500-505

3. Koditschek, D. E.: Exact robot navigation by means of potential functions:Some topological considerations. Proceedings of the IEEE International Conference on Robotics and Automation (1987) 1-6

4. Connolly, C. I., Burns, J. B., Weiss, R.: Path planning using Laplace's equation. Proceedings of the IEEE International Conference on Robotics and Automation (1990) 2102-2106.

5. Akishita, S., Kawamura, S., Hayashi, K.: Laplace potential for moving obstacle avoidance and approach of a mobile robot. Japan-USA Symposium on flexible automation, A Pacific rim conference (1990) 139-142.

6. Weinstock, R.: Calculus of variations with applications to physics and engineering. Dover Publications, Inc., New York (1974).

7. Zachmanoglou, E. C., Thoe, D. W.: Introduction to partial differential equations with applications. Dover Publications, Inc., New York (1986). 